\title{
Influence of flatness on structural strength of end spherical bulkhead
}

\author{
Jingyi Xiong, Yong Liu and Jianjun Ma \\ Wuhan second Ship Design and Research Institute, Wuhan, 430064, China \\ csic_xjy@163.com
}

\begin{abstract}
Keywords: spherical bulkhead, flatness, stress strength, ultimate load-bearing capacity, initial deflection

Abstract. The spherical bulkhead structure in style of sphere-toroid-cylinder combined shells is taken as the research object, and its structural strength with different "flatness" is studied. In order to characterize the geometric flat shape of the spherical bulkhead, the concept of "flatness" is put forward preliminary. Systematic numerical simulations of spherical bulkheads with different flatness are performed, and the effects of flatness on stress strength and ultimate bearing capacity are analyzed. The results show that stress on the inner-surface of the toroidal transition is significant with relatively small flatness, and the plastic deformation tends to soon occur and rapidly develop in the toroidal transition until the structure fails. And when the flatness is large (about larger than 0.7), there is high-level bending stress in the spherical shells and the ultimate bearing pressure is affected significantly by the initial deflection. The range of the flatness is suggested to be $0.35 \sim 0.55$. The conclusion of this paper could provide a reference for the design of spherical-toroid-cylinder combined shells structure.
\end{abstract}

\section{Introduction}

End spherical bulkhead is an important part of under vehicle pressure hulls and requires adequate structural strength to withstand external pressure. Compared with the plane bulkhead, the spherical bulkhead has advantages of high space utilization, light weight and so on. It is widely used in deep-dive vehicles. Spherical bulkhead structure generally consists of spherical shells, transition ring shells and cylindrical (conical) shells [1].

There have been published openly studies on the structural strength of end spherical bulkheads. Due to the symmetry of the structure, elastic foundation beam method and transfer matrix method (semi-theoretical method) are often used in the calculation of stress strength of spherical bulkheads, which can consider the inconsistency of neutral plane in the thick and thin junction [2], discontinuities of the slopes [3] and variable thickness. The elastic foundation beam method provides the theoretical basis for the structural design rule of the spherical bulkhead [4]. The influence of the main parameters of the spherical bulkhead on its stress strength can be analyzed expediently by using the finite element method [5]. The results $[3,5]$ show that the smooth toroidal transition can significantly alleviate the stress strength of the transition. Therefore, this paper focuses on the sphere-toroid-cylinder spherical bulkhead and its stress strength characteristics are further studied. In the aspect of spherical shells ultimate bearing capacity, in theory, the local defects of spherical shell are regarded as the independent shallow spherical shells which are restrained by the rest of the spherical shell [6, 7], and then shell theory is used to solve the problem. Besides, the influence of local dent defects [8], eigenvalue buckling mode defects $[8,9]$ and roundness deviation of the spherical shell $[10]$ on the bearing capacity was studied by means of finite element method, and some influence rules were obtained. However, the bearing capacity of sphere-toroid-cylinder spherical bulkhead structure has not been studied in detail.

In this paper, the structural strength of sphere-toroid-cylinder spherical bulkhead is analyzed. Firstly, the concept of spherical bulkhead's "flatness" is proposed preliminary, which can characterize the geometrical shape and mechanical properties of spherical bulkhead. On this basis, the effect of 
flatness on spherical bulkhead stress strength and the ultimate load-carrying capacity as well as the failure mechanism are studied with the consideration of the initial deflection.

\section{"Flatness" of Spherical Bulkhead and its Influence on Stress Strength}

\section{Stress Distribution Characteristics of Spherical Bulkhead}

The structural style of spherical bulkhead model structure is shown in Figure 1. The geometrical parameters in the model are followed: the radius of the spherical shell $R=1340 \mathrm{~mm}$, the radius of the toroidal transition $r=340 \mathrm{~mm}$, the cylindrical shell radius $R_{0}=1100 \mathrm{~mm}$, the toroidal transition axial length $H_{1}=221 \mathrm{~mm}$ and the spherical shell axial $H_{2}=469 \mathrm{~mm}\left(H_{1}\right.$ and $H_{2}$ change with $R$ and $\left.r\right)$, the spherical bulkhead length $H$ (the sum of $H_{1}$ and $H_{2}$ ), and the bulkhead thickness $t=16 \mathrm{~mm}$. Points A and $\mathrm{B}$ are the connection points of cylinder-toroid and sphere-toroid respectively. The elastic model $E$ $=1.96 \times 10^{5} \mathrm{MPa}$, the Poisson's ratio $\mu=0.3$, and the uniform external pressure $P_{0}=3 \mathrm{MPa}$. The fixed supported boundary condition acts on the end of ring-stiffened cylindrical shells. The cylindrical shell with four ribs in the model is used to eliminate the boundary effect on the spherical bulkhead structure.

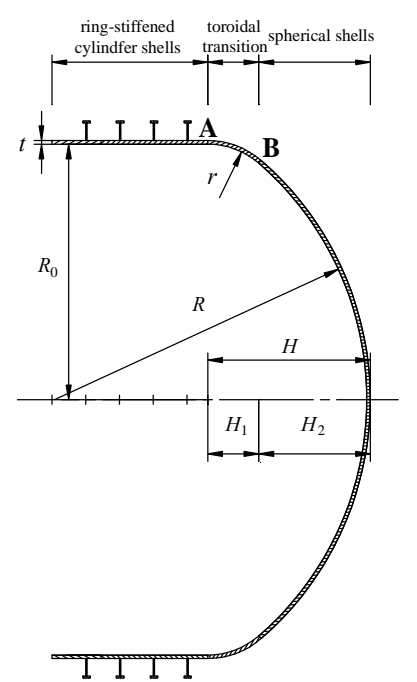

Fig.1 The sketch of sphere-toroid-cylinder spherical bulkhead

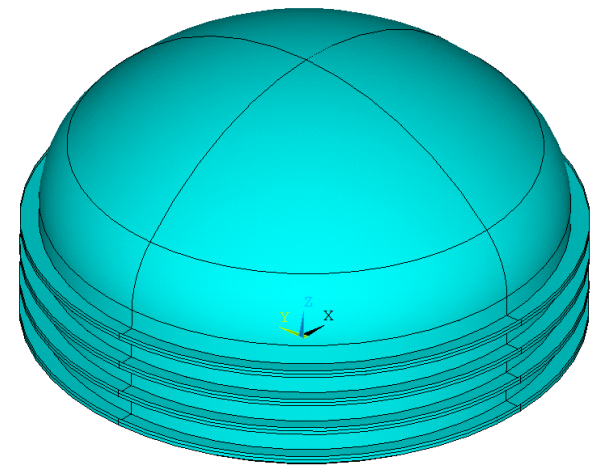

Fig.2 The geometric model of spherical bulkhead

The geometrical model of the spherical bulkhead is shown in Fig.2. The finite element software is used to calculate the stress strength. The stress distributions along the generatrix of spherical bulkhead (starting at the end of the cylindrical shell and ending at the vertex of the spherical shell) are as shown in Fig.3.

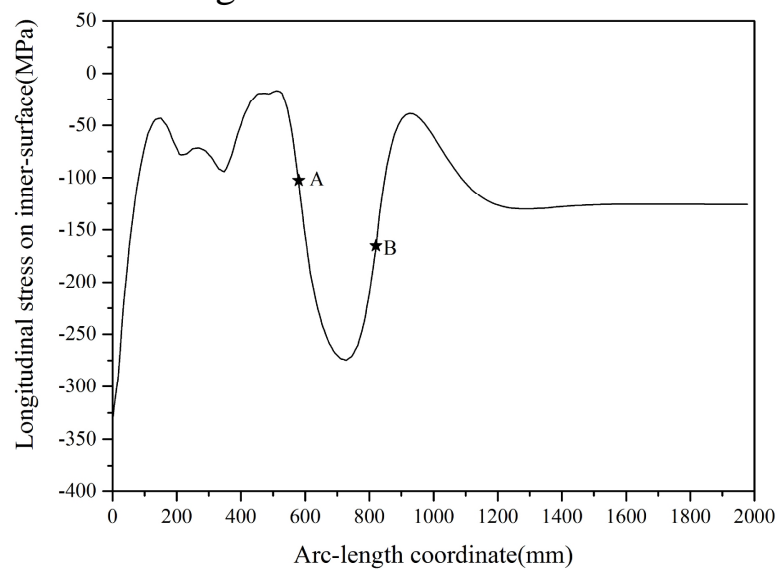

(a)

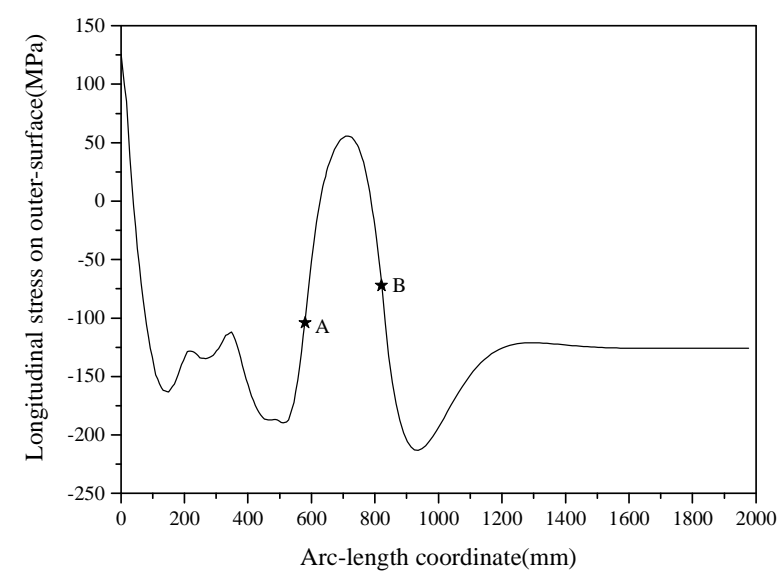

(b)

Fig.3Longitudinal stress distribution on surface 
The stress distributions have some characteristics as followed: the spherical shell zone far away from the transition is in the state of membrane stress; the longitudinal compressive stress is in a high level on the inner-surface of the toroidal transition (Fig. 3 (a)); there is large longitudinal compressive stress on the outer-surface of spherical shell near the transition (Fig. 3 (b)). The longitudinal compressive stresses of these two points should be investigated as focus.

\section{Concept of "Flatness"}

Studies[11] have shown that the stress state is more ideal when $R$ is smaller, $r$ is larger, that is, when the bulkhead axial length $H$ is larger (the maximum is equal to $R_{0}$, at the same time, the spherical shell and the toroidal transition become hemispherical shell structure). In engineering applications, due to the overall layout of the cabin, space utilization and other factors, $H$ is generally subjected to certain constraints. How to coordinate the size of the toroidal transition and the spherical shells to make the overall stress level lower, is worthy of discussion. For the sphere-toroid-cylinder type spherical bulkhead structure, the concept of "flatness" is proposed to be the ratio between $H_{1}$ and $H$, which is used to reflect the combination relationship between toroidal transition and spherical shells. The flatness is represented by $F$,namely

$$
F=H_{1} / H
$$

The flatness $F$ can visually describe the geometric characteristics of spherical bulkheads. The larger the $F$ is, the longer $H_{1}$ relatively is, the larger $R$ and $r$ are, the more flat and abundant the spherical bulkhead appears to be, as shown in Figure 4.

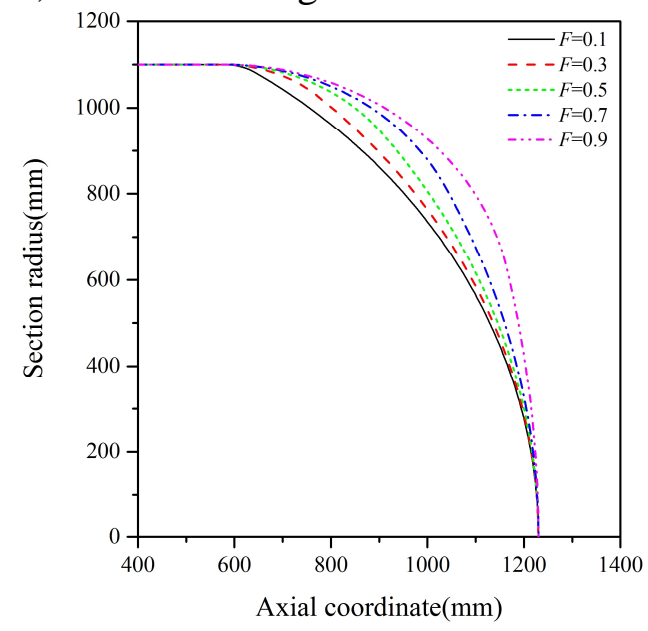

Fig.4 The shape of spherical bulkhead varying flatness $F$

The $F$ can also characterize the mechanical characristics of spherical bulkheads. When $F$ is smaller, the spherical shell part is larger, and the region in membrane stress state is larger, the stress distribution is more similar to that of the complete spherical shell (only in the short toroidal transition region, due to the sharp transition, Stress changes dramatically and stress amplitude is great). When $F$ is larger, the bending moment and the region which bending moment act on in the spherical shell are larger, and the bending stress get to be the main stress component. As $F$ tends to 1, the spherical shell tends to the plane, and the stress distribution in spherical shell region is similar to that of the plane bulkhead (without horizontal girders and stiffeners).

\section{Effect of Flatness on Stress Strength of Spherical Bulkhead}

In order to study the influence of the flatness on the stress strength of the spherical bulkhead structure, this paper initially takes $H=650 \mathrm{~mm}, F$ takes $0.1,0.2,0.3,0.4,0.5,0.6,0.7$ respectively. 


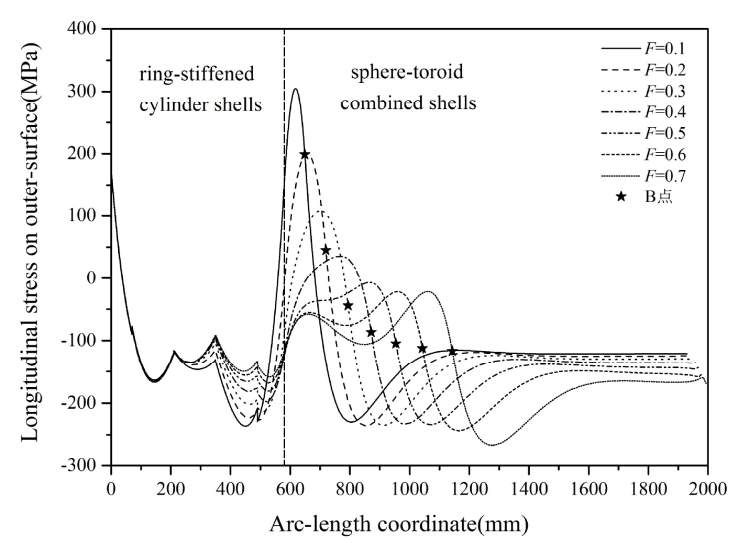

(a)

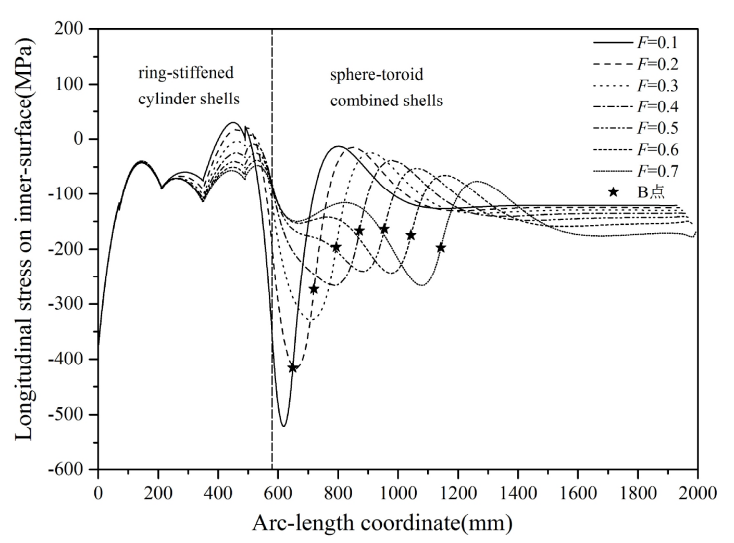

(b)

Fig. 5Longitudinal stress distribution on surface varying flatness

The stress distribution on surface with different flatness is shown in Fig.5, when the $F$ is smaller, the longitudinal compressive stress on the inner-surface is larger and the tensile stress could be high on the outer-surface due to the large longitudinal bending moment in the middle of the toroidal transition. When the $F$ increases, the longitudinal bending moment and the longitudinal compressive stress on the inner surface will reduce significantly. If the $F$ is too large, the longitudinal bending moment and the longitudinal stress will increase instead. And the region of membrane stress state on the spherical shells will be small, which is not conducive to structural arrangements (such as openings).

In order to verify the widespread influence of flatness under different $H$, four high-level state components of stress and internal force are selected as the reference to further analysis: the maximum compressive stress $\sigma_{\max 1}$ on inner-surface of the toroidal transition, the longitudinal stress $\sigma_{\max 2}$ on outer-surface of the toroidal transition, the maximum longitudinal stress $\sigma_{\max 3}$ on outer-surface of spherical shells and the maximum longitudinal bending moment $M_{1}$ in the toroidal transition. $H$ takes $500 \mathrm{~mm}, 550 \mathrm{~mm}, 600 \mathrm{~mm}, 650 \mathrm{~mm}, 700 \mathrm{~mm}, 750 \mathrm{~mm}, 800 \mathrm{mmrespectively} \mathrm{to} \mathrm{research} \mathrm{the} \mathrm{changes} \mathrm{of}$ state components above with $F$, as shown in Figure 6. (The bending moments per unit length of the section in Figure 6 (d), in $\mathrm{kN}$, the same below)

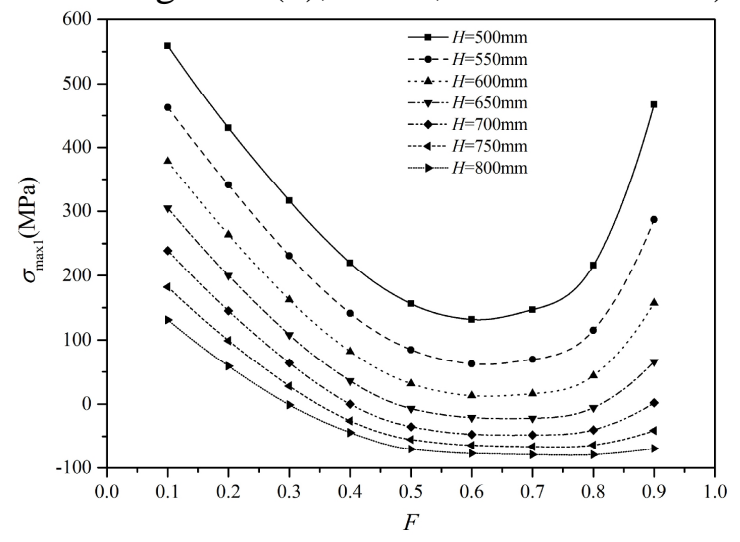

(a)

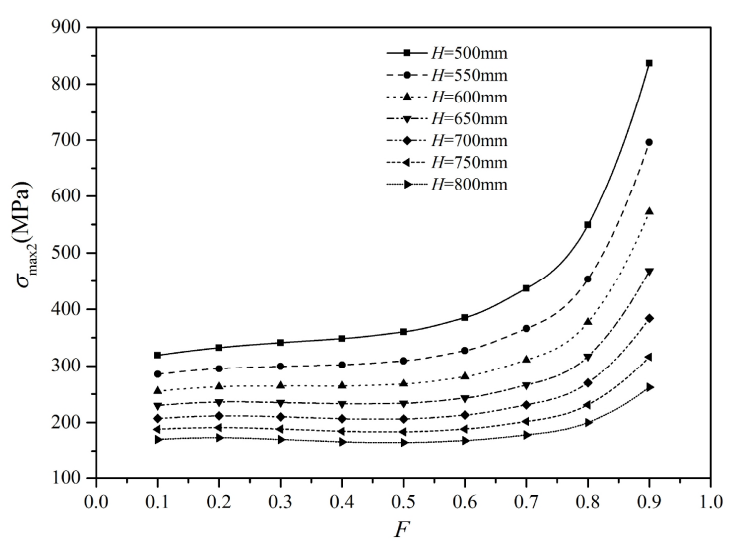

(b) 


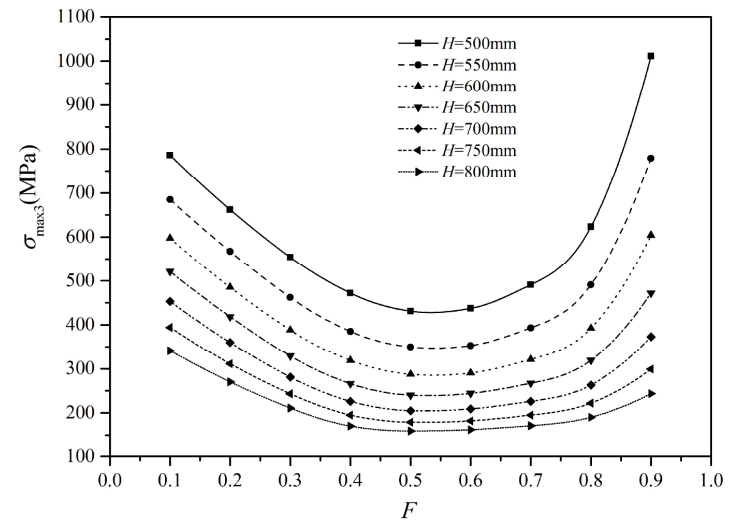

(c)

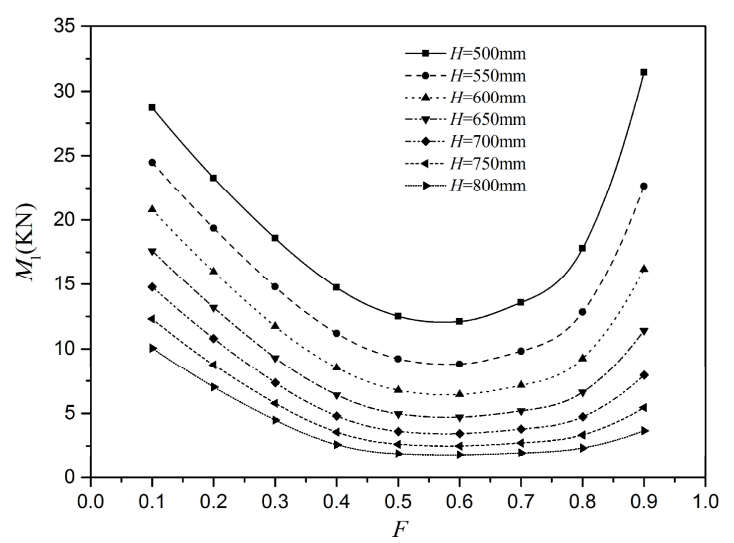

(d)

Fig.6 Four conference items change with flatness varying $\mathrm{H}$

The influence of $F$ on the stress state is basically the same under different $H$ : when the $F$ is too small, $\sigma_{\max 1}$ and $\sigma_{\max 2}$ will be very large due to large-scale $M_{1}$; when the $F$ is too large, they will increases instead. Therefore, moderate $F$ makes the stress level lower and stress distribution gentler.

In addition, the smaller the $H$, the higher sensitivity of the impact of $F$ on the stress amplitude; in the same $F$, the greater the $H$ is, the lower the stress level and bending moment are. Briefly, the overall arrangement conditions permitting, Larger $H$ is better.

\section{Influence of Flatness on Ultimate Bearing Capacity}

\section{Calculation model}

The spherical bulkhead model is shown in Fig.2. Both material and Geometric nonlinearity have been taken into consideration in the calculation of ultimate bearing capacity of spherical bulkhead. The ideal elastic-plastic model is adopted for the materials of the whole structure. The material yield criterion is Von Mises criterion. Considering the geometrical nonlinearity (large deformation) of the structure, the initial deflection is introduced into the model, and the arc length method is used in the nonlinear calculations.

The forms of initial deflection include the local dent form, the eigenvalue-buckling mode defect form, the true deflection form of objects or models. The true deflection form has a greater randomness due to the manufacturing process and eigenvalue buckling modal defect forms generally are the most unfavorable to the structure [10]. Consequently, the eigenvalue buckling mode is adopted as the initial deflection form in this paper. Taking into account the limits of shape deviation in engineering construction, the initial deflection amplitude $f$ does not exceed $0.6 t$ ( $t$ is the thickness) in this study.

Influence of Flatness on Ultimate Bearing Capacity of Spherical Bulkhead Structure with

\section{Initial Deflection}

In order to study the effect of flatness $F$ on the ultimate bearing capacity of spherical bulkhead structure, $F$ is taken as $0.1 \sim 0.8$. The ultimate bearing pressure $P$ of spherical bulkhead under each amplitude initial deflection $f$ is calculated.

Table.1 Ultimate bearing pressure $P$ varying flatness $F$ and amplitude of initial deflection $f$

\begin{tabular}{cccccccccccccc}
\hline$F$ & \multicolumn{10}{c}{$f$} \\
\cline { 2 - 14 }$y$ & 0.01 & 0.05 & 0.10 & 0.15 & 0.20 & 0.25 & 0.30 & 0.35 & 0.40 & 0.45 & 0.50 & 0.55 & 0.60 \\
\hline 0.1 & 0.4533 & 0.4532 & 0.4529 & 0.4523 & 0.4515 & 0.4500 & 0.4458 & 0.4312 & 0.4112 & 0.3925 & 0.3762 & 0.3590 & 0.3411 \\
0.2 & 0.4776 & 0.4775 & 0.4770 & 0.4769 & 0.4749 & 0.4702 & 0.4584 & 0.4378 & 0.4100 & 0.3857 & 0.3667 & 0.3436 & 0.3285 \\
0.3 & 0.5093 & 0.5087 & 0.5069 & 0.5044 & 0.4853 & 0.4550 & 0.4174 & 0.4127 & 0.3891 & 0.3692 & 0.3430 & 0.3287 & 0.3196 \\
0.4 & 0.5496 & 0.5474 & 0.5458 & 0.5282 & 0.4844 & 0.4585 & 0.4255 & 0.3955 & 0.3733 & 0.3452 & 0.3211 & 0.3107 & 0.3027 \\
0.5 & 0.5801 & 0.5748 & 0.5540 & 0.5004 & 0.4647 & 0.4245 & 0.3994 & 0.3562 & 0.3465 & 0.3100 & 0.3054 & 0.2987 & 0.2924 \\
0.6 & 0.5564 & 0.5496 & 0.5135 & 0.4608 & 0.4141 & 0.3884 & 0.3538 & 0.3357 & 0.3003 & 0.2903 & 0.2832 & 0.2764 & 0.2697 \\
0.7 & 0.5269 & 0.5163 & 0.4829 & 0.4353 & 0.3830 & 0.3336 & 0.3270 & 0.2976 & 0.2816 & 0.2664 & 0.2537 & 0.2433 & 0.2351 \\
0.8 & 0.4142 & 0.3904 & 0.3544 & 0.3239 & 0.2957 & 0.2701 & 0.2488 & 0.2345 & 0.2210 & 0.2126 & 0.2031 & 0.1989 & 0.1955 \\
\hline
\end{tabular}


Note: in the table.1, $H=700 \mathrm{~mm}$, the initial deflection amplitude $f$ is the ratio between the actual amplitude and shell thickness, and the ultimate bearing pressure $P$ is under dimensionless treatment as the ratio between the actual damage pressure value and a certain reference pressure value.

The results of the above table are plotted as the curves of the ultimate bearing pressure changing with the amplitude of the initial deflection $\mathrm{f}$ at different flatness F, as shown in Fig.7

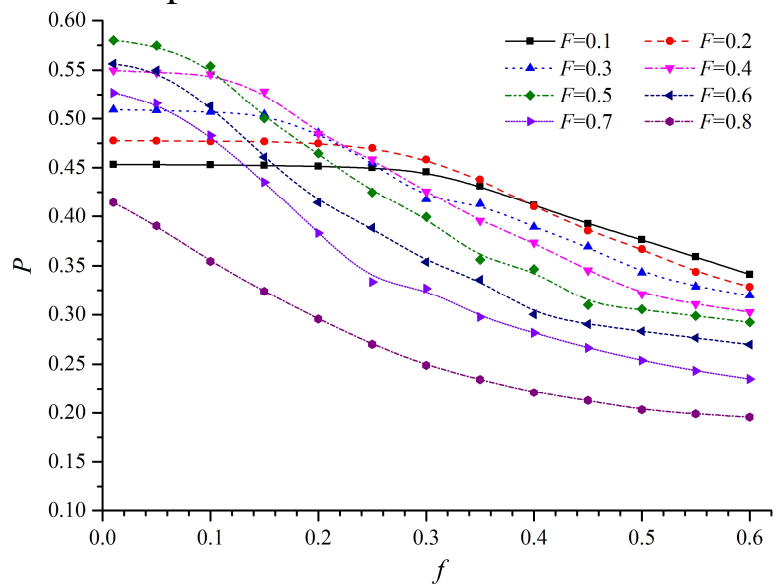

(a)

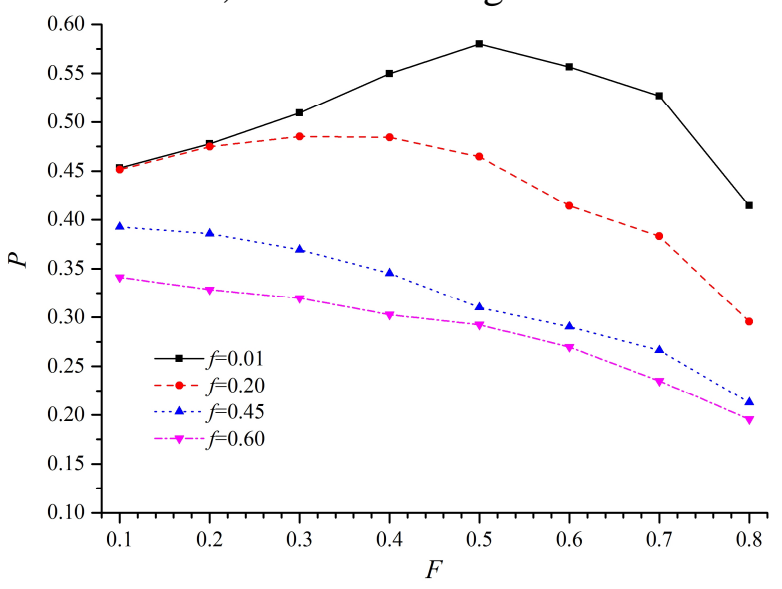

(b)

Fig.7 Ultimate bearing capacity with different $F$ and $f$

The structural ultimate bearing pressure $P$ decreases with the increase of the initial deflection amplitude $f$. When the $F$ is small, the change of the $P$ with the $f$ is gentler, especially when the $f$ is less than 0.2. When the $F$ is larger, the change of the $P$ with the $f$ is more significant, that is, $P$ is more sensitive to the $f$. In addition, when the $f$ is small (about less than 0.20), with the increase of $F$, the $P$ increases and then decreases. When $f$ is greater (about greater than 0.45 ), the $P$ will decrease with the increase of the $F$.

For more intuitive clarity of the impact of $F$ on $P$ under different $f$, the contour diagrams are shown in Figure 8 (a) (c) below. It can be seen from the figure that when $F$ is 0.35-0.6 and the $f$ is small, the failure pressure gets larger value (corresponding to the red area in the contour). When $\mathrm{F}$ is larger, the gradient of the cloud is larger, that is, $P$ changes significantly with $f$. When $H$ are $600 \mathrm{~mm}$ and $800 \mathrm{~mm}$, the distribution patterns of contours are similar to that of $H=700 \mathrm{~mm}$, that is, the effect of $F$ on the ultimate bearing capacity of spherical bulkhead with initial deflection has the general rule. When $F$ is about $0.35 \sim 0.55$, the spherical bulkhead structure has a higher ultimate load-carrying capacity in the range of smaller initial deflection amplitude $f$ (less than about $0.3 \mathrm{t}$ ). From Fig. 8 (d), when the $H$ is $600 \mathrm{~mm}, 700 \mathrm{~mm}, 800 \mathrm{~mm}$, the response surface of ultimate bearing pressure $P$ is distributed upward sequentially, which means the bigger the $H$ is, the stronger the bearing capacity is.

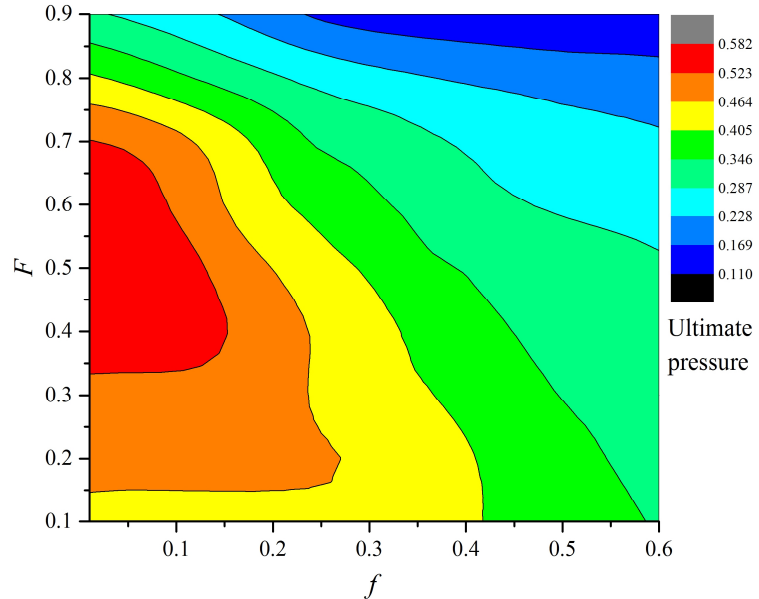

(a)

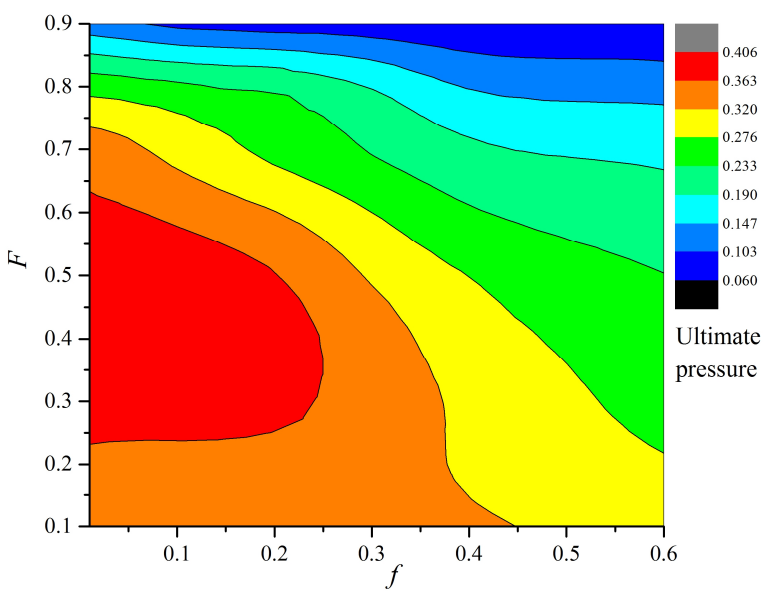

(b) 


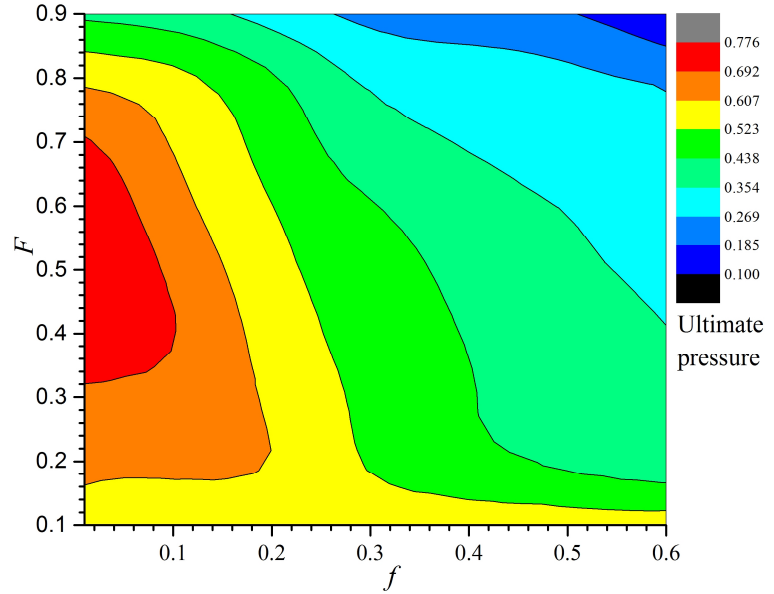

(c)

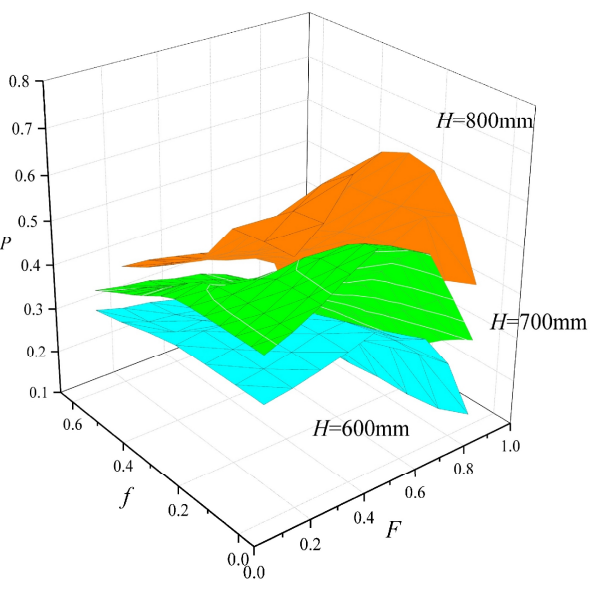

(d)

Fig.8 Contours of ultimate bearing pressure with different $H$

\section{Plastic Extension and Failure Mechanism Analysis}

To study the failure modes and mechanism of spherical flat bulkheads with different flatness, several models with typical flatness are selected for analysis.

When the flatness $F$ is 0.1 , toroidal transition acute and rapid, the inner surface stress level is high. Consequently, plastic deformation occurs first inside the surface of toroidal transition, as shown in Fig. 9(a).With the load increasing, the plastic deformation gradually extends to the outer surface of the toroidal transition, and then the plastic flows without limit until the spherical bulkheads reach the ultimate failure pressure. In the unloading phase, the large deformation occurs in toroidal transition which has been completely in a plastic state and plastic deformation quickly flows to the sphere-toroid junction and adjacent sphere region, where the partial depressions appear.

When the $F$ is 0.4 , as shown in 9(b), plastic deformation also occurs first inside the surface of toroidal transition, but has not been fully developed; when the load continues to increase, the plastic deformation appear in the spherical shell region defects located, and develop rapidly.

When the $F$ is 0.7 , the toroidal transition section is relatively large and the bending stress there is smaller. However, there is high-level bending stress in the spherical shell region (Fig. 9(c)). The plastic deformation also occurs first on outer-surface of the initial deflection region of the spherical shell, and gradually spreads to the inner-surface and the surrounding region of the spherical shell. In the unloading phase, the concave deformation of the deflection region is further increased, so that the inner surface of the middle parts between the adjacent depressed region are squeezed to form plastic hinge line, resulting in great plastic strain until crushing and tearing.

In summary, when the flatness is small, plastic deformation tend to soon occur and rapidly develop in the toroidal transition, and the ultimate bearing pressure is small relatively; when the flatness is too large, there is high-level bending stress in the spherical shell region, and the ultimate bearing pressure is affected significantly by the initial deflection. When the flatness is moderate, the toroidal transition and spherical shells could be balanced appropriately, and the entire structure will not appear plastic collapse damage rapidly, making the carrying capacity higher.

(a)

$F=0.1$

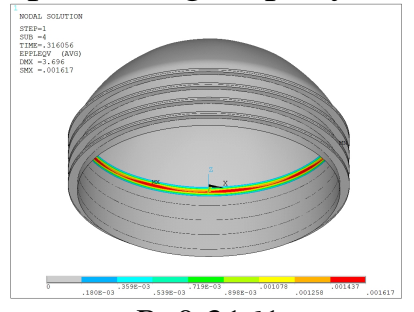

$P=0.3161$

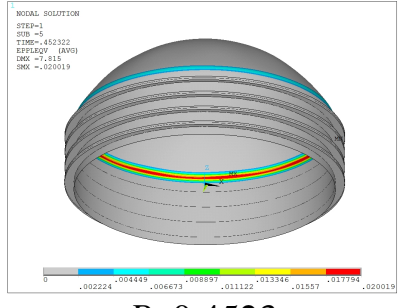

$P=0.4523$

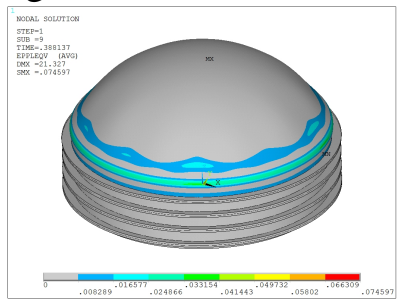

$P=0.3881$

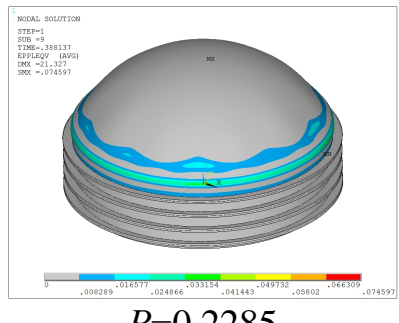

$P=0.2285$ 
(b)

$F=0.4$

(c)

$F=0.7$

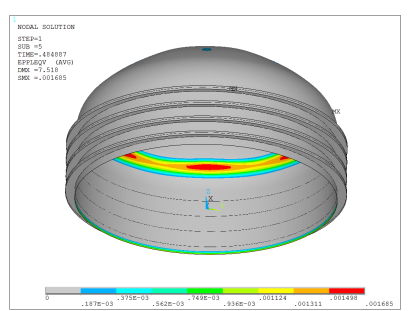

$P=0.4849$

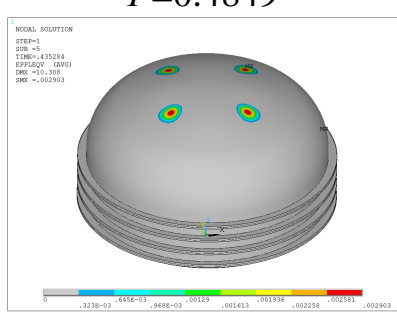

$P=0.4353$

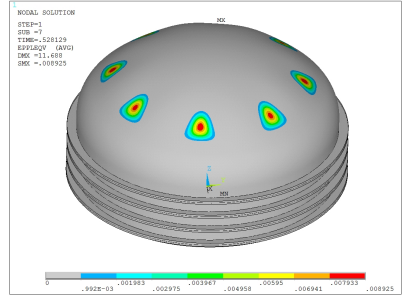

$P=0.5281$

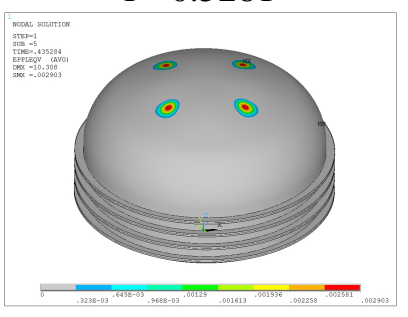

$P=0.2451$

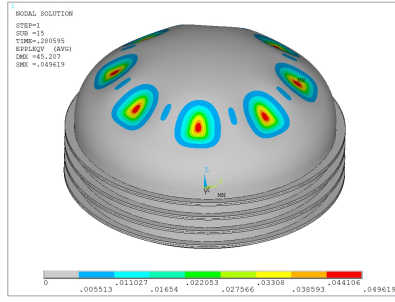

$P=0.2806$

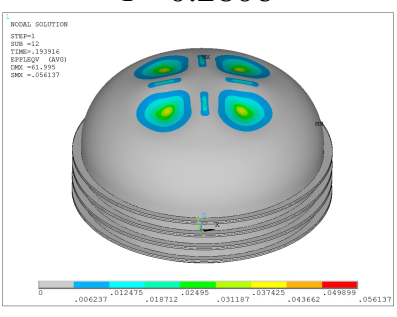

$P=0.1939$

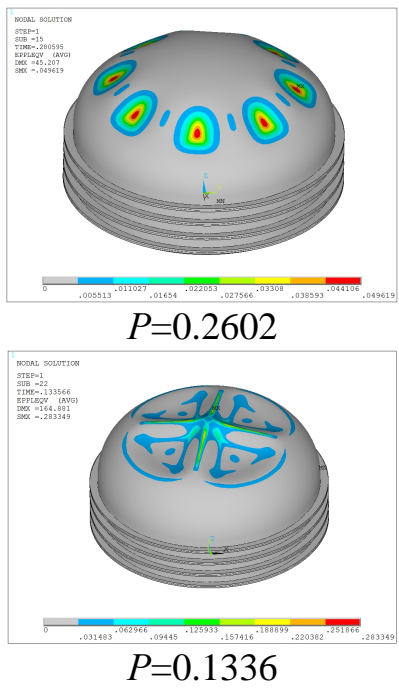

Fig.9 Plastic extension and failure mode

\section{Conclusion}

The structural strength of the sphere-toroid-cylinder type spherical bulkhead is analyzed in this paper. The concept of "flatness" is preliminary; and effects of flatness on the stress strength and ultimate load carrying capacity of spherical bulkhead structure are studied. The following conclusions are obtained:

(1) The preliminary proposed concept of "flatness" can characterize the geometry form and mechanical characteristics of the spherical bulkhead structure. When the flatness is small, the spherical bulkhead appears relatively deep, the longitudinal stress is larger on the inner-surface of the toroidal transition. When the flatness is too large, the bulkheads appear to be flat and abundant, and there is bending stress in the spherical shells, reducing the capacity of membrane stress against external pressure.

(2) The flatness has certain influence on the ultimate bearing capacity of the spherical bulkhead structure: when the flatness is small, the ultimate bearing pressure changes little with the initial deflection amplitude, and the plastic strain appears and develop in the toroidal transition until the structure fails; When the flatness is too large, the bending stress is more prominent due to the disturbance of the initial deflection, which leads to plastic strain occurring and expanding rapidly in the initial deflection region.

(3) Combining the influence of the flatness on the stress strength and ultimate bearing capacity of the spherical bulkheads, the range of the flatness is suggested to be $0.35 \sim 0.55$.

\section{References}

[1] Xu Jiping: Submarine strength[M] (National Defense Industry Press, 1987) (In Chinese)

[2] Zhu Bangjun, Wang Dan, WAN Zhengquan: An analytical solution for stresses of the end spherical bulkhead. Journal of Ship Mechanics, 2011, 15(11), 1255-1263. (In Chinese)

[3] HUANG Ni, XIA Fei, YANG YUhua, WAN Peng. A effect study on the curved angle in toroid-cone of the sphere-toroid-cone combined shells[J]. SHIP SCIENCE AND TECHNOLOGY, 2011,33(10): 25-28. (In Chinese)

[4] GJB/Z21A-2001.Method for design and calculation of submarine structure [S]. Beijing: COSTIND, 2001. (In Chinese) 
[5] FAN Ming-qi, WANG Yong-jun,LIU Xin. Analysis of dome for submarine structure[J]. Journal of Ship Mechanics, 2007,11(4):594-599. (In Chinese)

[6] Zheng Yanshuang, LuZhengfuand, ZhangDingwu. Collapse Pressure of Spherical Shells with Initial Imperfections under Uniform Pressure[J]. SHIPBUILDING OF CHINA,1986(1): 48-58. (In Chinese)

[7] Li Tianyun, Zheng Yanshuang. Nonlinear Stability Analysis of Spherical Shell with Imperfection under Uniform External Pressure[J]. J. Huazhong Univ. of Sci. \& Tech.1997,25(11):90-92 (In Chinese)

[8] WANG Zi-li, WANG Ren-hua, YU Ming-hua, LILiang-bi.The Influence of the Initial Imperfections on theUltimateStrength of Manned Deep-sea Submersible Pressure Sphere Hull[J]. SHIPBUILDING OF CHINA. 2007, 48(2): 45-50. (In Chinese)

[9] LU Bei, LIU Tao, CUI Wei-cheng.Ultimate strength of pressure spherical hull in deep-sea manned submersibles[J].Journal of Ship Mechanics,2004, 8(1): 51-58. (In Chinese)

[10] Huang Ni. Strength and Stability Analyses forSphere-Toroid-Cone Rotational Shells[D]. Wuhan: China Ship Development \& Design Center, 2012. (In Chinese)

[11] WANG Ren-hua, YUMing-hua, LI Liang-bi, WANG Zi-li.Influence of initial deflection on plastic stability ofmanneddeep-sea submersible's pressure sphere hull[J]. THE OCEAN ENGINEERING,2006, 23(4): 111-115. (In Chinese) 